\title{
Frequency of "incidental" serous tubal intraepithelial carcinoma (STIC) in women without a history of or genetic risk factor for high-grade serous carcinoma: A six-year study is $^{2}$
}

\author{
Emily E.K. Meserve ${ }^{\mathrm{a}}$, Jelena Mirkovic ${ }^{\mathrm{e}}$, James R. Conner ${ }^{\mathrm{b}}$, Eric Yang ${ }^{\mathrm{c}}$, Michael G. Muto ${ }^{\mathrm{d}}$, Neil Horowitz ${ }^{\mathrm{d}}$, \\ Kyle C. Strickland ${ }^{a}$, Brooke E. Howitt ${ }^{a}$, Christopher P. Crum ${ }^{\mathrm{a}, *}$ \\ a Division of Women's E Perinatal Pathology, Department of Pathology, Brigham and Women's Hospital, 75 Francis Street, Boston, MA 02115, USA \\ ${ }^{\mathrm{b}}$ Department of Pathology and Laboratory Medicine, Mt. Sinai Hospital, 600 University Avenue Toronto, ON M5G 1X5, Canada \\ c Department of Pathology, Stanford University Medical Center, 300 Pasteur Drive, Stanford, CA 94305, USA \\ d Division of Gynecologic Oncology, Department of Obstetrics \&' Gynecology, Brigham and Women's Hospital, 75 Francis Street, Boston, MA 02115, USA \\ e Department of Pathology, Sunnybrook Health Sciences Centre, 2075 Bayview Ave. Toronto, Ontario, M4N 3M5, Canada
}

\section{H I G H L I G H T S}

- "Incidental" serous tubal intraepithelial carcinomas (STIC) are uncommon (1/873).

- Most incidental STICs are discovered during surgery for other epithelial neoplasms.

- Many incidental STICs are found in women with endometrioid neoplasia.

- Further studies are needed to clarify the frequency of STIC and its relevance to HGSC.

\section{A R T I C L E I N F O}

\section{Article history:}

Received 10 January 2017

Received in revised form 19 April 2017

Accepted 21 April 2017

Available online 4 May 2017

\section{Keywords:}

Serous carcinoma

Fallopian tube

Serous tubal intraepithelial carcinoma

\section{A B S T R A C T}

\section{Objective}

The purpose of this study was to determine the prevalence of incidentally discovered serous tubal intraepithelial carcinoma in women without a genetic risk for or history of high grade serous carcinoma (HGSC) in the gynecologic tract.

Methods. All pathology reports at our institution that included bilateral salpingectomies from January 2006December 2011 were examined in women $>50$ years old in which the entire tube or the distal one-third was examined histologically with the complete (proximal and distal fallopian tube) or modified (distal one third of the tube) SEE-FIM protocol. Cases were divided into: Group 1, a history of or known risk factors (BRCA1 or BRCA2 mutations) for HGSC and Group 2, those without these attributes for whom a STIC would be unexpected (incidental). Women undergoing unspecified "risk-reducing" procedures were included in Group 1.

Results. Of 4051 identified total, 2268 had complete examination of the distal fallopian tube and were age 50 or above. Of these, 1747 were in group 2 . Two STICs were identified $(0.1 \%)$, one associated with a grade 2 endometrial endometrioid adenocarcinoma and one with a low-grade ovarian serous carcinoma in the setting of a serous borderline tumor.

Conclusions. Incidental STICs in women over age 50 are uncommon. However, the significance of lesser tubal atypias ( $0.3 \%$ in this study), risk of STIC in women with no epithelial pathology and the risk imposed by coexisting endometrioid neoplasia are unclear and require further study.

(c) 2017 Elsevier Inc. All rights reserved.

\footnotetext{
in Supported by a grant from the Department of Defense (OC130500 to CPC).

* Corresponding author at: Department of Pathology, Brigham and Women's Hospital, Boston, MA 02115, USA.

E-mail address: ccrum@partners.org (C.P. Crum).
}

\section{Introduction}

Extra-uterine high grade serous carcinoma (HGSC) is the most common variant of pelvic cancer in women and over $95 \%$ of patients present with high stage (II or greater) disease, including the long-term survivors [1]. As a consequence, attention is now heavily focused on therapies and interventions aimed at prevention. Due to research on early serous 
carcinogenesis in the last $10-15$ years, many cases of HGSC are now believed to arise from the distal fallopian tube in the form of serous tubal intraepithelial carcinoma (STIC) [2-5]. Thus, in addition to prophylactic salpingo-oophorectomy, which is a powerful risk-reducing intervention for women with germ line BRCA1 or BRCA2 mutations, $[6,7][8,9]$ the Society of Gynecologic Oncology (2013) and American College of Obstetricians and Gynecologists (2015) have suggested that opportunistic salpingectomy be considered at the time of hysterectomy for "averagerisk" (alternatively "low-risk") women after the completion of child bearing to reduce the risk of a later HGSC $[10,11]$. The success of opportunistic salpingectomy is predicated on the idea that this surgery will prevent the development of an intraepithelial precursor to HGSC or intercept a precursor before spread outside of the tube.

To have an impact on serous cancer incidence, opportunistic salpingectomy theoretically either removes precursors that would have progressed to HGSC or, by removing the fallopian tubes, preempts the tubal serous carcinogenic sequence entirely. Germane to both goals is the prevalence of STICs in women who are not considered at high risk for HGSC. The prevalence has not been established in large studies but at institutions where complete microscopic examination of the distal fallopian tube has been implemented (SEE-FIM protocol), it is estimated that the frequency of STIC in these women is between 0.6 and $1.1 \%$ [12$16]$.

The purpose of this study was to determine the frequency of STICs discovered incidentally in women not considered at high risk for HGSC and whose entire distal fallopian tubes had been submitted for pathologic examination. The study population encompassed a 6 year span from 2005 to 2011.

\section{Materials \& methods}

This study was approved by the Institutional Review Board at Brigham and Women's Hospital. Anatomic pathology reports during the period January 2006 to December 2011 were screened to identify all cases with, at minimum, bilateral salpingectomies from women who were $>50$ years old $(n=4051)$. This age cut-off was selected 1$)$ to reflect a conservative estimate of the completion of childbearing and 2 ) to collect patients at a time when risk of HGSC begins to increase while excluding young women at extremely low-risk of HGSC.

Surgical pathology reports were manually reviewed for gross descriptions with documentation of the presence of the fallopian tubes and/or fimbriae and microscopic keys with sections specifically designated as entirely submitted fimbriae, distal one-third of the fallopian tube, or fallopian tube. Cases were retained in the study if bilateral distal fallopian tubes were grossly identified and entirely submitted for microscopic examination $(n=2286)$. Unilateral salpingectomy cases were excluded, even if the fallopian tube was completely examined, as absence of STIC in the contralateral fallopian tube could not be documented. Diagnoses were collected from surgical pathology reports and were used to classify cases into one of five categories: 1) benign (leiomyoma, uterine prolapse, etc.), 2) endometrioid neoplasia (of endometrium or ovary) including endometrial intraepithelial neoplasia, 3) HGSC of the endometrium, adnexa, or peritoneum, 4) other non-HGSC neoplasms (including low grade serous carcinoma), and 5) prophylactic procedures for highrisk women (RRS or RRSO).

For the purposes of this study, The subjects were divided into two groups. Group 1 consisted of categories 3 and 5 above, women at risk for HGSC or who had been diagnosed with HGSC. Group 2 consisted of groups 1,2, and 4 . In these patients the presence of STIC would normally be unexpected or "incidental".

All diagnoses of STIC and tubal epithelial atypia for which a diagnosis of STIC was considered but excluded were identified from pathology reports. A diagnosis of STIC required 1) a multilayered non-ciliated epithelium, 2) increased nuclear to cytoplasmic ratio, 3) loss of cell polarity with irregularly arranged nuclei in the vertical plane. Strong or completely absent p53 staining was present and the proliferative (MIB-
1) index was increased, albeit variably. Additionally, tubal/fimbrial sections from $5 \%$ of women without a diagnosis of STIC were re-reviewed to confirm the original pathologic interpretation.

\section{Results}

\subsection{Study population}

During the 6-year study period, 4051 cases including bilateral salpingectomies from women $>50$ years of age were identified; 2268 cases were over age 50 and had documented complete gross and microscopic examination of the distal fallopian tube and were retained in the study. Of these, 1747 (77.0\%) cases were in Group 2 and had no history of risk for or a diagnosis of HGSC [Fig. 1].

STIC is only rarely encountered as an "incidental" finding in women without the presence of or risk for HGSC.

Eighty STICs were encountered in this study, 78 of which were identified either from risk reducing salpingo-oophorectomies or cases with advanced HGSC. Two STIC were identified incidentally in 1747 women in Group 2 (0.1 One was associated with a low grade serous carcinoma of the ovary and coexisting bilateral borderline serous tumors and the other with a grade 2 endometrial endometrioid adenocarcinoma. [Fig. 2].

The clinical management and follow-up of both LRW found to have incidental STIC during the six year study period was dictated by the concurrent neoplastic process (ie. low grade serous carcinoma or endometrial endometrioid adenocarcinoma). [Table 1] In neither case was an elevated CA125 documented prior to surgery.

\section{Discussion}

Beginning in 2000, a progressive accumulation of data has implicated the distal fallopian tube as a site of origin of HGSC by identification of precursors in tubal epithelium [2-5]. As a consequence, in addition to clinical management guidelines recommending salpingo-oophorectomy for women with germ-line mutations in BRCA1 or BRCA2, recommendations have been published for opportunistic salpingectomy as a cancer preventive in women who are not at increased risk for ovarian cancer [9-11].

Beginning in January of 2005, we instituted the SEE-FIM protocol at Brigham and Women's Hospital, based on the hypothesis that the distal fallopian tube was the most vulnerable site for the onset of precursors to HGSC [12]. Later that year, Cass and colleagues reported that most fallopian tube carcinomas arose in or near the fimbria [17]. Subsequently, Medeiros et al. and Callahan et al. reported that virtually all incidentally discovered HGSCs in RRSOs from women at genetic risk were found in the distal fallopian tube, and Kindelberger et al. showed that over $40 \%$ of women with HGSC had evidence of STIC in their distal fallopian tube $[3,12,18]$. Thus, in our practice, the SEE-FIM protocol or a protocol in which the entire distal fallopian tube was submitted is followed in all salpingectomy specimens.

The purpose of this study was to assess the prevalence of STIC in a setting where they are detected "incidentally", i.e. in specimens from patients where there was no expectation of an increased detection risk. In assessing the statistical information we emphasize several potential variables that would influence detection rate in this population. First, in every case in this study ( $n=1747)$ at least the distal one-third of the fallopian tube was examined, often but not always with the entire proximal tube. It is conceivable that expansion of the sectioning protocol to include the entire proximal two-thirds of the fallopian tube segments in every case might have increased the odds of identifying incidental STICs. However, we emphasize that the likelihood of uncovering a significant number of STICs in the proximal segments would be low. Second, tubes were not exhaustively sectioned to uncover small lesions that might escape notice with routine sectioning. We have found that exhaustive sectioning of "negative" fallopian tubes from women with HGSC will uncover a STIC in 6\% (Soong R, unpublished data). This will 


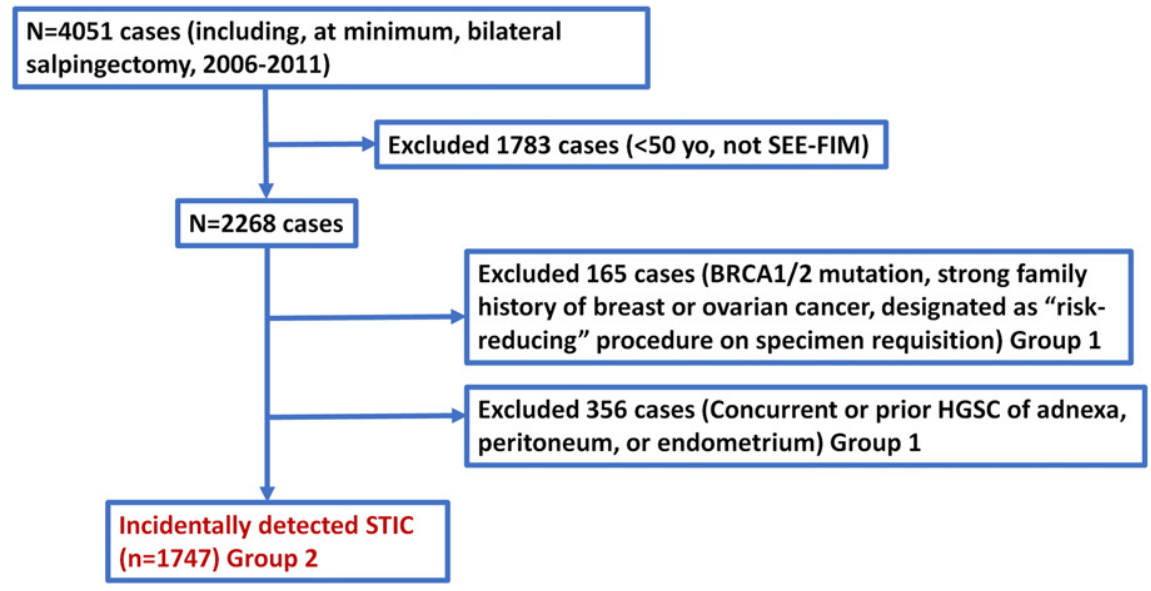

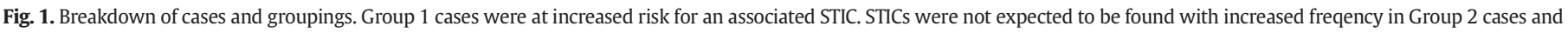
were designated as "incidental" in these patients.

slightly increase the overall percentage with STIC but only a few percentage points. Third, we excluded women under age 50 years from the study for three reasons: 1) women under age 50 with no known increased risk have a very low likelihood of either HGSC or STIC and are effectively a different population in terms of risk, 2) in our consultation practice where we have received 52 cases of STIC or atypias for review in approximately 10 years; 10 were under age 50 and 6 of these were found to have mutations in BRCA1 or BRCA2, and thus would not have
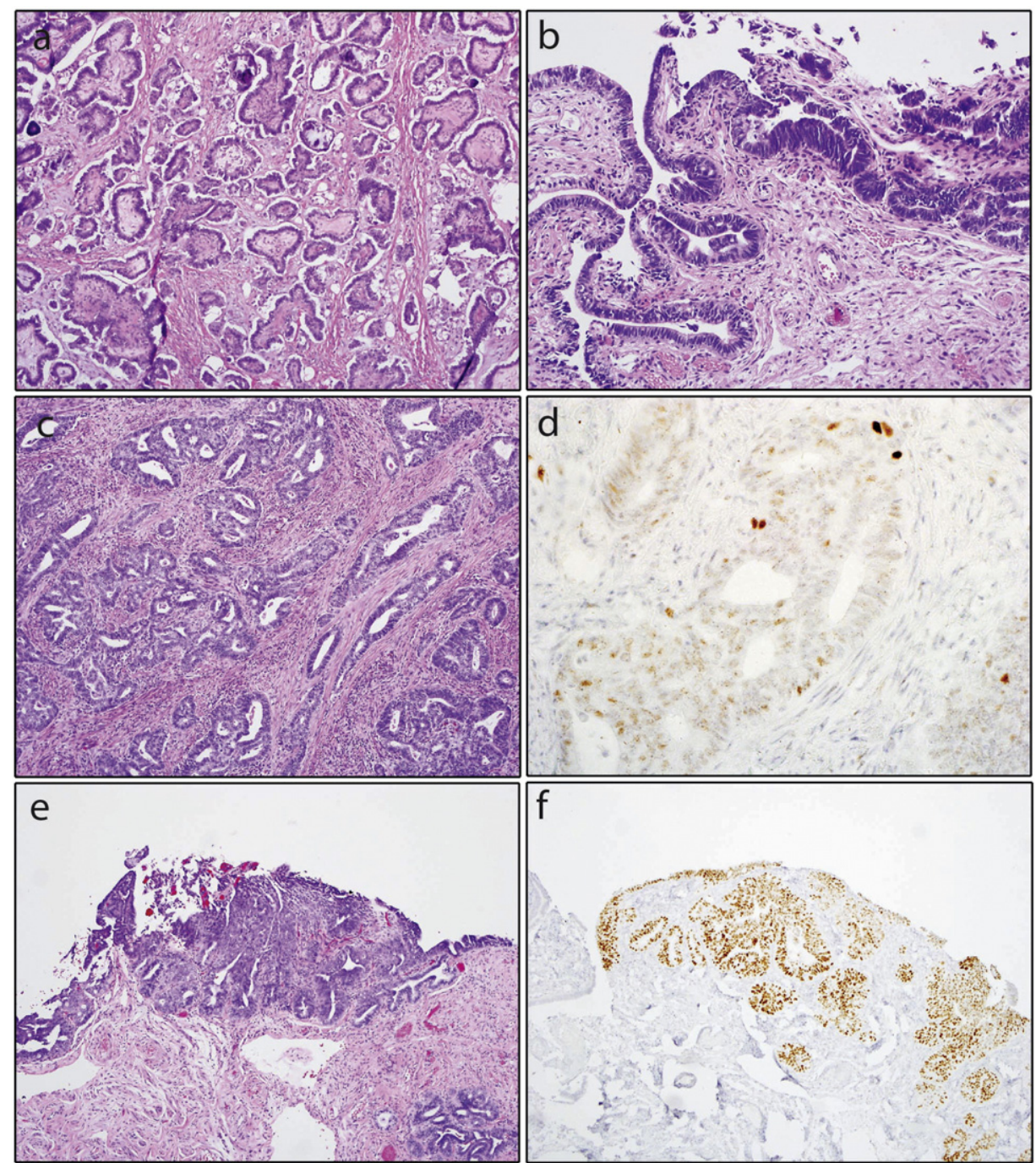

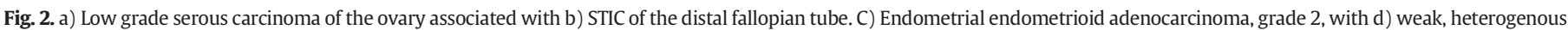

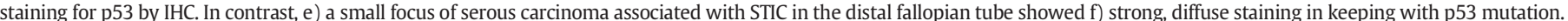


Table 1

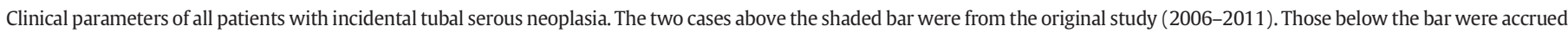
later (2012-2015).

\begin{tabular}{|c|l|l|l|l|l|}
\hline Age & Indication & Concurrent diagnosis & Serous lesion & $\begin{array}{l}\text { Peritoneal } \\
\text { cytology }\end{array}$ & Follow-up \\
\hline 51 & $\begin{array}{l}\text { Ovarian } \\
\text { masses }\end{array}$ & $\begin{array}{l}\text { Low-grade ovarian } \\
\text { serous carcinoma }\end{array}$ & STIC & Negative & 24 months NED \\
\hline 72 & EMB: EMCA & G1 EMCA & STIC & Negative & 42 months NED \\
\hline 67 & EMB: EMCA & G1 EMCA & STIC & None & None \\
\hline & & & $\begin{array}{l}\text { STIC } \\
\text { HGSC on ovary } \\
\text { (stage Ila) }\end{array}$ & $\begin{array}{l}\text { Suspicious for } \\
\text { carcinoma }\end{array}$ & $\begin{array}{l}28 \text { months } \\
\text { NED. }\end{array}$ \\
\hline 71 & EMB: EMCA & G1 EMCA & $\begin{array}{l}\text { R\&L STIC, HGSC on } \\
\text { ovaries (stage Illa) }\end{array}$ & $\begin{array}{l}\text { Positive } \\
\text { (carcinoma) }\end{array}$ & $\begin{array}{l}10 \text { months } \\
\text { NED. }\end{array}$ \\
\hline 64 & Pelvic mass & $\begin{array}{l}\text { Mucinous } \\
\text { cystadenoma }\end{array}$ & STIC & Negative & 5 months NED \\
\hline
\end{tabular}

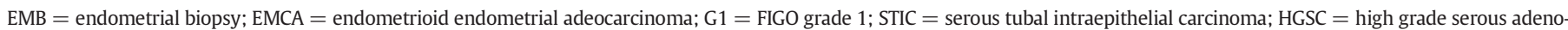
carcinoma; NED = no evidence of disease.

been included in the study group, and 3) in our surgical practice, no STIC has yet to be discovered in a women under age 50 who were not at high risk.

This study comprehensively examines the yield of 6 years of this protocol and represents the largest examined cohort of women who were not deemed high risk. The frequency of STIC in this population is estimated to be one per every 873 cases, which is lower than estimates from all previously published studies. We also performed a preliminary analysis of cases accessioned between January 2012 and December 2015 from women age 50 or over including bilateral salpingectomies by searching our pathology database and identified an additional 4 STICs. Based on the total case volume in this interval we estimate these 4 STICs occurred in an estimated cohort of approximately 1150 or 1 per 287 cases. The associated pathology included a mucinous cystadenoma, mucinous cystadenofibroma, and two cases of endometrial endometrioid adenocarcinoma. The estimated frequency of STICs and the circumstances under which they were discovered in this second group make two important points. First, the frequency will vary as a function of the time frames involved. Second, all of the cases of STIC identified in this study in women in group 2 were undergoing staging procedures for ovarian masses or concurrent epithelial neoplasia. None were identified in women who would fall into the group commonly assumed to have undergone "opportunistic" salpingectomy during a procedure for benign conditions. The absence of incidental STICs in women undergoing surgery for indications other than suspected neoplasia might be explained in part by two variables: 1) women who underwent morcellation procedures in which tubes could not be optimally examined and were excluded, and 2) referral bias, with a gynecologic surgery practice heavily weighted toward suspected oncology cases. Still, the frequency of incidental STIC in these women appears particularly low and remains to be further defined. Still, the question remains as to what percentage of women without any gynecologic epithelial neoplasia that can be expected to develop a tubal precursor. A related question is whether lesser atypias containing p53 mutations that do not fulfill the criteria for STIC $(6 / 1747$ or $0.3 \%$ from 2006 to 2011 in this study) impose any longitudinal risk of HGSC later in life.

The second observation (with caveat) was the finding of STICs in women with concurrent endometrioid neoplasia. Concurrent endometrioid and serous tumors are presumed to arise via independent molecular pathways and cells of origin $[5,19]$. Coexistence of these two tumor types is considered rare, confirmed in this study where STIC was discovered in only $0.2 \%$ of endometrioid neoplasms. However, other studies have suggested an association between STIC and endometrioid neoplasia $[16,20]$. Moreover, we have identified an association, albeit unclear, between benign proliferations with a "clonal" appearance (stem cell outgrowths (SCOUTs)) in the tube - some with endometrioid histology - and HGSC [21,22]. Thus it is conceivable that a small but unique subset of women have a vulnerability to both endometrioid and serous cancer precursor pathways or that both pathways share some yet to be clarified biologic underpinning. It does not appear to be via a mismatch repair defect based on the normal findings in two of the tumors.

In summary, the frequency of "incidentally" discovered STIC in is low in postmenopausal women, particularly in women with no gynecologic epithelial neoplasms. The extent to which this will inform our understanding of the efficacy of opportunistic salpingectomy will hinge on a clearer vision of how the serous carcinogenic sequence develops and the percentage of HGSCs that can be attributed to STICs.

\section{Conflict of interest}

The authors have no conflicts of interest.

\section{References}

[1] F. Dao, B.A. Schlappe, J. Tseng, J. Lester, A.M. Nick, S.K. Lutgendorf, S. McMeekin, R.L Coleman, K.N. Moore, B.Y. Karlan, A.K. Sood, D.A. Levine, Characteristics of 10-year survivors of high-grade serous ovarian carcinoma, Gynecol. Oncol. 141 (2016) 260-263.

[2] J.M. Piek, P.J. van Diest, R.P. Zweemer, J.W. Jansen, R.J. Poort-Keesom, F.H. Menko, J.J Gille, A.P. Jongsma, G. Pals, P. Kenemans, R.H. Verheijen, Dysplastic changes in prophylactically removed fallopian tubes of women predisposed to developing ovarian cancer, J. Pathol. 195 (2001) 451-456.

[3] D.W. Kindelberger, Y. Lee, A. Miron, M.S. Hirsch, C. Feltmate, F. Medeiros, M.J. Callahan, E.O. Garner, R.W. Gordon, C. Birch, R.S. Berkowitz, M.G. Muto, C.P. Crum, Intraepithelial carcinoma of the fimbria and pelvic serous carcinoma: evidence for a causal relationship, Am. J. Surg. Pathol. 31 (2007) 161-169.

[4] Y. Lee, A. Miron, R. Drapkin, M.R. Nucci, F. Medeiros, A. Saleemuddin, J. Garber, C. Birch, H. Mou, R.W. Gordon, D.W. Cramer, F.D. McKeon, C.P. Crum, A candidate precursor to serous carcinoma that originates in the distal fallopian tube, J. Pathol. 211 (2007) 26-35.

[5] R.J. Kurman, M. Shih Ie, The origin and pathogenesis of epithelial ovarian cancer: a proposed unifying theory, Am. J. Surg. Pathol. 34 (2010) 433-443.

[6] J.S. Berek, E. Chalas, M. Edelson, D.H. Moore, W.M. Burke, W.A. Cliby, A. Berchuck, Society of Gynecologic Oncologists Clinical Practice C: prophylactic and risk-reducing bilateral salpingo-oophorectomy: recommendations based on risk of ovarian cancer, Obstet. Gynecol. 116 (2010) 733-743.

[7] L.P. Shulman, Hereditary breast and ovarian cancer (HBOC): clinical features and counseling for BRCA1 and BRCA2, Lynch syndrome, Cowden syndrome, and LiFraumeni syndrome, Obstet. Gynecol. Clin. N. Am. 37 (2010) 109-133 (Table of Contents.).

[8] N.D. Kauff, J.M. Satagopan, M.E. Robson, L. Scheuer, M. Hensley, C.A. Hudis, N.A. Ellis, J. Boyd, P.I. Borgen, R.R. Barakat, L. Norton, M. Castiel, K. Nafa, K. Offit, Risk-reducing salpingo-oophorectomy in women with a BRCA1 or BRCA2 mutation, N. Engl. J. Med. 346 (2002) 1609-1615.

[9] M.B. Daly, C.W. Dresher, M.S. Yates, J.M. Jeter, B.Y. Karlan, D.S. Alberts, K.H. Lu, Salpingectomy as a means to reduce ovarian cancer risk, Cancer Prev. Res. (Phila.) 8 (2015) 342-348. 
[10] J.L. Walker, C.B. Powell, L.M. Chen, J. Carter, V.L. Bae Jump, L.P. Parker, M.E. Borowsky, R.K. Gibb, Society of Gynecologic Oncology recommendations for the prevention of ovarian cancer, Cancer 121 (2015) 2108-2120.

[11] Committee on Gynecologic P, Committee opinion no. 620: salpingectomy for ovarian cancer prevention, Obstet. Gynecol. 125 (2015) 279-281.

[12] F. Medeiros, M.G. Muto, Y. Lee, J.A. Elvin, M.J. Callahan, C. Feltmate, J.E. Garber, D.W. Cramer, C.P. Crum, The tubal fimbria is a preferred site for early adenocarcinoma in women with familial ovarian cancer syndrome, Am. J. Surg. Pathol. 30 (2006) 230-236.

[13] N. Koc, S. Ayas, L. Uygur, The association of serous tubal intraepithelial carcinoma with gynecologic pathologies and its role in pelvic serous cancer, Gynecol. Oncol. 134 (2014) 486-491.

[14] P.A. Shaw, M. Rouzbahman, E.S. Pizer, M. Pintilie, H. Begley, Candidate serous cance precursors in fallopian tube epithelium of BRCA1/2 mutation carriers, Mod. Pathol. 22 (2009) 1133-1138.

[15] J.T. Rabban, K. Garg, B. Crawford, L.M. Chen, C.J. Zaloudek, Early detection of highgrade tubal serous carcinoma in women at low risk for hereditary breast and ovarian cancer syndrome by systematic examination of fallopian tubes incidentally removed during benign surgery, Am. J. Surg. Pathol. 38 (2014) 729-742.

[16] J.D. Seidman, J. Krishnan, A. Yemelyanova, R. Vang, Incidental serous tubal intraepithelial carcinoma and non-neoplastic conditions of the fallopian tubes in grossly normal adnexa: a Clinicopathologic study of 388 completely embedded cases, Int. J. Gynecol. Pathol. (2015).

[17] I. Cass, C. Holschneider, N. Datta, D. Barbuto, A.E. Walts, B.Y. Karlan, BRCA-mutationassociated fallopian tube carcinoma: a distinct clinical phenotype? Obstet. Gynecol. 106 (2005) 1327-1334.

[18] M.J. Callahan, C.P. Crum, F. Medeiros, D.W. Kindelberger, J.A. Elvin, J.E. Garber, C.M. Feltmate, R.S. Berkowitz, M.G. Muto, Primary fallopian tube malignancies in BRCApositive women undergoing surgery for ovarian cancer risk reduction, J. Clin. Oncol. 25 (2007) 3985-3990.

[19] M.A. Merritt, D.W. Cramer, Molecular pathogenesis of endometrial and ovarian cancer, Cancer Biomark 9 (2010) 287-305.

[20] J.C. Morrison, L.Z. Blanco Jr., R. Vang, B.M. Ronnett, Incidental serous tubal intraepithelial carcinoma and early invasive serous carcinoma in the nonprophylactic setting: analysis of a case series, Am. J. Surg. Pathol. 39 (2015) 442-453.

[21] E.Y. Chen, K. Mehra, M. Mehrad, G. Ning A. Miron, G.L. Mutter N. Monte, B.J. Quade, F.D. McKeon, Y. Yassin, W. Xian, C.P. Crum, Secretory cell outgrowth, PAX2 and serous carcinogenesis in the fallopian tube, J. Pathol. 222 (2010) 110-116.

[22] C.M. Quick, G. Ning, J. Bijron, A. Laury, T.S. Wei, E.Y. Chen, S.O. Vargas, R.A. Betensky, F.D. McKeon, W. Xian, C.P. Crum, PAX2-null secretory cell outgrowths in the oviduct and their relationship to pelvic serous cancer, Mod. Pathol. 25 (2012) 449-455. 